\title{
Interferometry in the focal plane with KERNEL
}

\author{
Frantz Martinache ${ }^{a}$ \\ ${ }^{a}$ Laboratoire Lagrange, Université Côte d'Azur, Observatoire de la Côte d'Azur, CNRS, Parc \\ Valrose, Bât. H. FIZEAU, 06108 Nice
}

\begin{abstract}
KERNEL is an ERC funded project (ERC CoG 2015 \# 683029) that aims at boosting the angular resolution of any telescope using the power of interferometry. Looking at the formation of astronomical images as the result of an interferometric process, the mathematical framework at the heart of the KERNEL project makes it possible to use direct focal images to extract either robust observables called kernel-phases that extend the self-calibrating properties of closure-phase to arbitrary apertures and do super-resolution; or wavefront metrology information that can easily and efficiently scale up to multiple references for wide field applications. Currently limited to the small aberration regime, the KERNEL framework can be more widely applied when dealing with spectrally dispersed focal data. The paper presents the current status of the KERNEL project and some of its recent developments and applications that lead to recommendations to reach the ELT's full angular resolution potential, as soon as the first generation AO system becomes available.
\end{abstract}

\section{INTRODUCTION}

Imaging in the high angular resolution regime comes down to solving the problem of deconvolving an unknown object function from an ever changing point spread function (PSF). The development of adaptive optics (AO), now ubiquitous on major ground based observatories, dramatically changed this arena, by turning previously seeing dominated images into telescope diffraction dominated ones, thus enabling high angular resolution observations on large telescopes. An 8-to-10 meter telescope observing in the H-band (1.6 $\mu \mathrm{m})$ exhibits a 0.04 arcsecond diffraction limit, sufficient to resolve Jupiter like planets in formation around stars of the nearby young associations Taurus (distance $\sim 145 \mathrm{pc}$ ) 122 The resolving power of an AO-equiped 30-meter class telescope should prove sufficient to image Super-Earth type planets in the habitable zone of the nearby M-dwarfs $\left.{ }^{3}\right]$

In an image dominated by the diffraction features of a telescope's aperture, the capacity to detect a structure in the direct neighborhood of a bright object will depend on how well the resulting instant PSF, affected by residual optical aberrations, is characterized. The ever changing nature of the PSF, simultaneously affected by the stochastic atmospheric turbulence and the partial correction provided by adaptive optics renders such deconvolution difficult. This is a constraint shared by another observing technique: optical stellar interferometry. Translated into the language of optical interferometry, the effective detection power will be driven by the uncertainty associated to the complex visibility measurements the aperture of the telescope gives access to. From a collection of calibrated complex visibilities acquired for several interferometric baselines, one is able to go back to a reliable description of the source observed, by virtue of a classical result known as the Van Cittert-Zernike theorem, that can be seen as an equivalent to the classical image-object convolution relation that one is used to invoke when describing diffraction-dominated images.

Looking at image formation as the result of an interferometric process is not just a fun cerebral exercise: interferometry led to the development of several clever calibration strategies that one can draw inspiration from. The only concession to make is that one must no longer directly look at images, but at their Fourier transform counterpart. Visibilities in the Fourier plane are complex numbers, whose amplitude and phase are usually considered separately, as in the example provided in Fig. 1 .

The phase is related to symmetry properties of the targeted object. For a centro-symmetric observed in ideal conditions, the recorded phase would alternate between only two values of 0 and 180 degrees. Intermediate values, such as the ones represented in the left panel of Fig. 1 are the sign that the object is asymmetric: this makes the phase part of the Fourier space a naturally good place to look for companions around bright stars.

email: frantz.martinache@oca.eu 

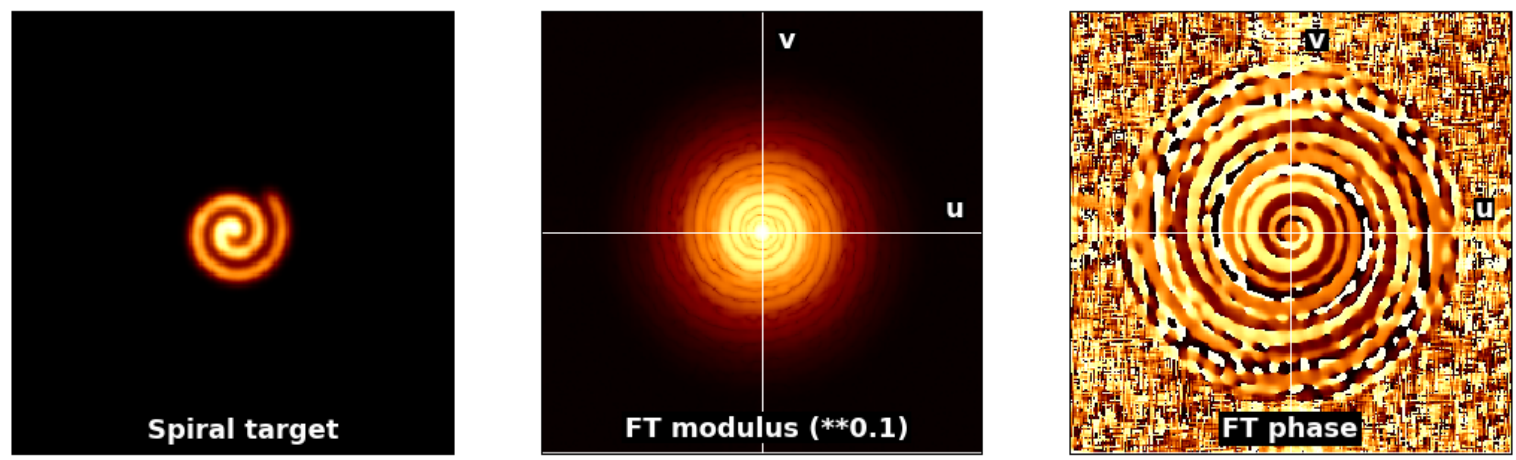

Figure 1. Example of the relation between a spiral object and its associated complex visibility, split into modulus and phase represented over the entire uv-plane.

Residual wavefront aberrations however also contribute to the phase measured in the Fourier plane. In the context of a two-telecope interferometer, the effect of aberration is seen (at first order) as a random atmospheric piston term that shifts fringes around and makes the phase $\Phi$ measured in the Fourier plane a degenerate function of both object phase $\Phi_{o}$ and instrumental piston $\Delta \varphi: \Phi=\Phi_{o}+\Delta \varphi$. Something interesting however occurs when the interferometer simultaneously collects fringes produced by at least three apertures: the instrumental pistoninduced degeneracy can be partly lifted when combining together phase measurements. The closure-phase ${ }^{4}$ is the simplest possible combination of instrumental phases: adding together the individually polluted phases measured along three baselines corresponding to a triangle of elementary apertures in the pupil produces a new observable (the closure-phase) that is insensitive to residual instrumental phase. This is a powerful postprocessing technique that is routinely exploited by optical long-baseline interferometry ${ }^{15}$ as well as with nonredundant aperture masking interferometry ${ }^{\sqrt{6}}$ to complete the image quality improvement provided by adaptive optics.

A single dish aperture gives access to a potentially very large number of interferometric baselines. One illustration of this is provided in Fig. 22 the real continuous aperture of a telescope can be represented as a discrete interferometric array of virtual sub-apertures arranged on a regular grid so as to reproduce the features of the continuous aperture like its outer diameter, central obstruction and spider arms. This particularly rich interferometer produces a very large number of interferometric baselines, visible in the left panel of Fig. 2. The interpretation of the information available in the phase part of the Fourier transform of diffraction dominated images affected by wavefront aberrations is however difficult.

The phase $\Phi^{j}$ associated to one of the short virtual baselines, typically near the center of the covered Fourier plane finds itself sampled several hundreds of times by the virtual array. Such a baseline is said to be $r^{j}$ times redundant. The general equation for the phase sampled at this one location is given by:

$$
\phi^{j}=\operatorname{Arg}\left(\sum_{k=1}^{r^{j}} v_{0}^{j} \exp i\left(\phi_{0}^{j}+\varphi_{k}\right)\right)
$$

where $v_{o}^{j}$ is the object visibility modulus for the baseline of index $j$ and $\varphi_{k}$ are the components of the pupil-phase aberration $\varphi$ that contaminate the information $\Phi_{0}$ sampled by this virtual baseline. By itself, this expression cannot be inverted: the phase measured at a specific location of the Fourier plane is that of a sum of random phasors, of unpredictable phase and amplitude. When in the image, one observes random speckles, in the Fourier plane, one observes random phase. Instrumental phase and information on the target are intertwined and the general deconvolution problem knows no easy solution.

This difficulty is what motivated the deployment of non-redundant aperture masks in the first place. With a physical aperture mask preventing the contributions of redundant baselines to mix in the Fourier plane, the extraction of unique object Fourier-phase information is straightforward. Yet the direct interpretation of the 

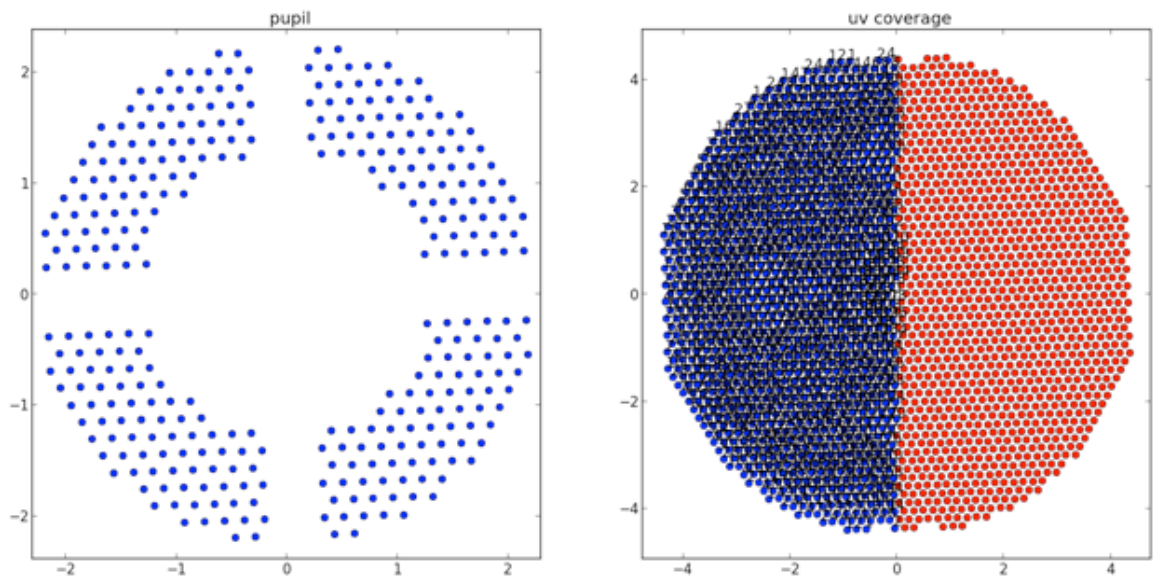

Figure 2. Left: Discrete interferometric representation of the continuous diffractive aperture of the Palomar Hale "Medium Cross" pupil mask of the PHARO instrument. Right: Resulting coverage of the Fourier plane by the virtual baselines associated to this model. A single aperture telescope can be compared to a very rich interferometer potentially offering access to a large number complex visibility measurements on a target of interest.

phase of in the Fourier plane without an aperture mask can also be drastically simplified when one measures the real impact of the partial correction provided by the use of adaptive optics. The goal of the KERNEL project is to exploit this possibility.

\section{THE KERNEL FRAMEWORK}

If the correction of the image by the adaptive optics suffices for a clear Airy pattern to form among speckles (wavefront RMS smaller than $\lambda / 4$ ), the instrumental phase residuals are of the order of $\sim 1$ radian or less. The complex phase equation for a selected virtual Fourier component (Eq. 1) can be drastically simplified via a linearization:

$$
\phi^{j} \approx \phi_{0}^{j}+\frac{1}{r^{j}} \sum_{k=1}^{r^{j}} \varphi_{k}
$$

The same equation can be written for all virtual Fourier components: the only difference is that to each virtual Fourier component, one will associate a different true object Fourier phase $\Phi_{o}^{j}$ and a different combination of the components of the pupil-phase aberration vector $\varphi$. The knowledge of the census of the virtual apertures contributing to all virtual Fourier components can be encapsulated in a single linear operator $\mathbf{A}$, such that the phase sampled at all relevant coordinates of the Fourier-plane results from:

$$
\Phi=\Phi_{o}+\mathbf{A} \cdot \varphi
$$

This linear model is a direct equivalent of the classical image-object convolution relation: $\mathbf{I}=\mathbf{O} \otimes \mathbf{P S F}$, only expressed in terms of the Fourier-phase, where $\Phi$ replaces the raw image $\mathbf{I}, \Phi_{o}$ the object function $\mathbf{O}$ and $\varphi$ the PSF.

The geometric properties of the true aperture of the telescope will affect the algebraic properties of $\mathbf{A}$, such as its size and its rank. For the example highlighted in Fig. 2, the phase-transfer matrix $\mathbf{A}$ is a $1124 \times 331$ rectangular matrix, describing the way the 331-component pupil aberration vector $\varphi$ translate into a 1124-component Fourier phase spurious signature, according to the model of Eq. 3. A singular value decomposition of $\mathbf{A}$ is used to further characterize its properties: 


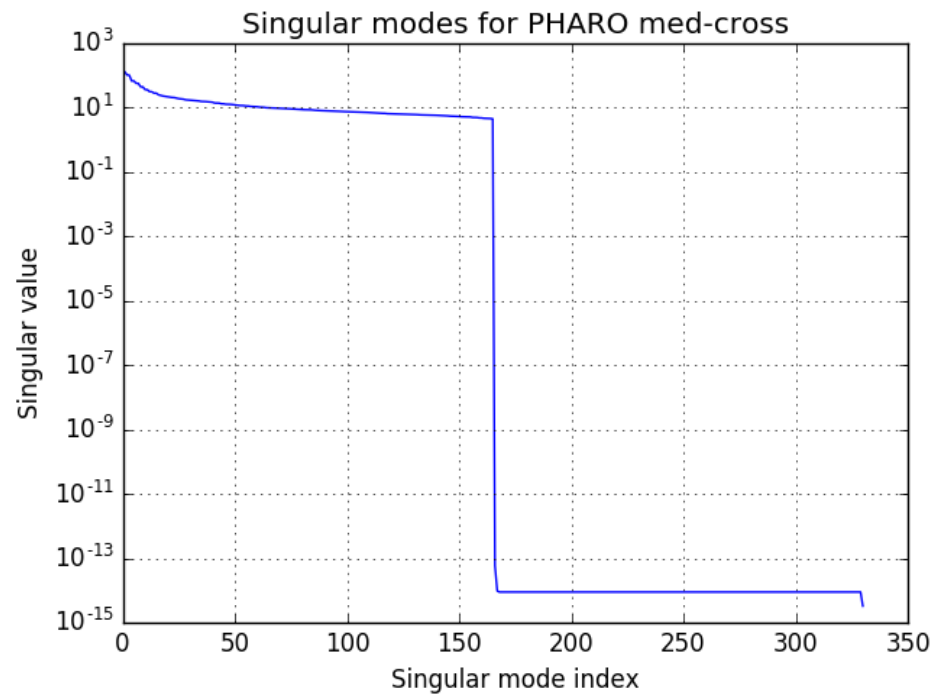

Figure 3. Singular values of the phase transfer matrix for the PHARO aperture model introduced previously. For this model, the first 166 singular values are strictly positive. After an obvious drop, the 166 remaining ones are at the level of numerical noise.
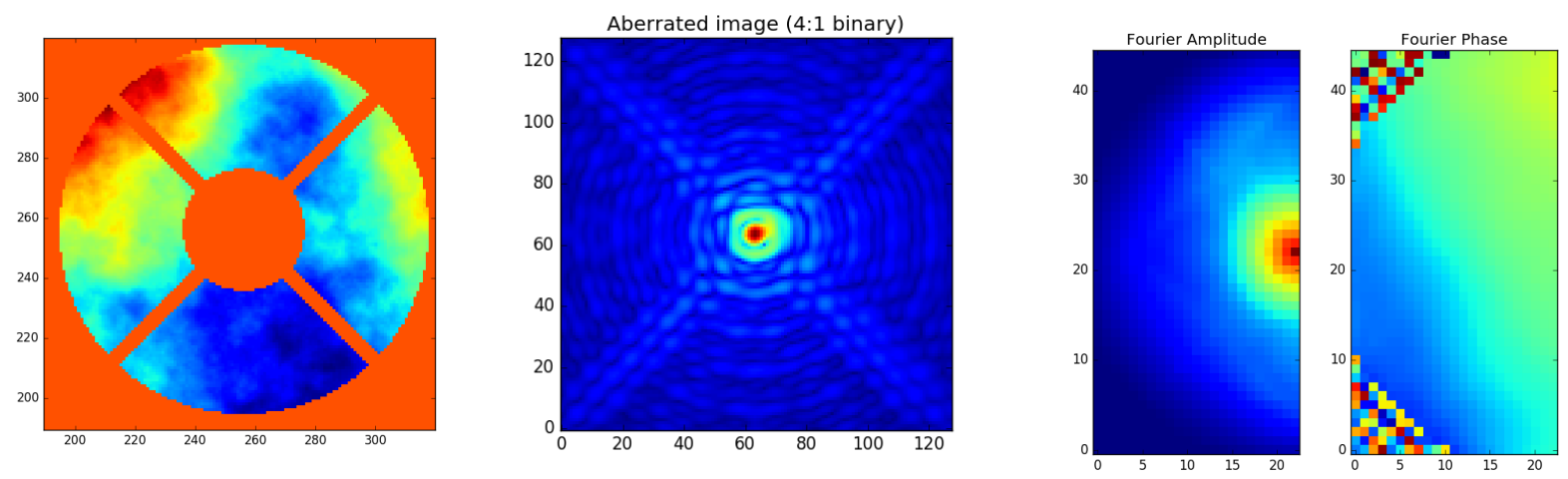

Figure 4. Left: Simulated Kolmogorov structured phase screen with a $140 \mathrm{~nm}$ RMS projected on the aperture of an 8-meter telescope. Middle: resulting simulated image produced on a 4:1 binary star system for an angular resolution smaller than the formal angular resolution $\lambda / D$. Right: representation of the Fourier transform (amplitude and phase) of this aberrated image.

$$
\mathbf{A}=\mathbf{U} \cdot \boldsymbol{\Sigma} \cdot \mathbf{V}^{T},
$$

where $\mathbf{U}$ and $\mathbf{V}^{T}$ are unitary matrices and $\boldsymbol{\Sigma}$ a diagonal matrix containing the singular values of $\mathbf{A}$. These singular values are real, positive and come out of the analysis sorted in decreasing order until they exactly reach zero at a location that will depend on the geometry of the aperture, as represented in Fig. 3 for the PHARO discrete aperture model introduced in Fig. 2. The plot features two distinct regions of strict positive and strict zero singular values: the rightmost part associated to zero singular values, suggests the existence of a sub-space of the Fourier-phase that is not affected by wavefront aberrations. The complementary sub-space associated to strictly positive singular values can be used for wavefront sensing purposes. The columns of $\mathbf{U}$ associated to zero singular values can be gathered into a new operator $\mathbf{K}$ that verifies $\mathbf{K} \cdot \mathbf{A}=\mathbf{0}$. This operator, corresponding to the left-hand null space (or kernel) of the phase transfer matrix $\mathbf{A}$ offers a generalized collection of closure-phase like quantities henceforth called kernel-phases. 

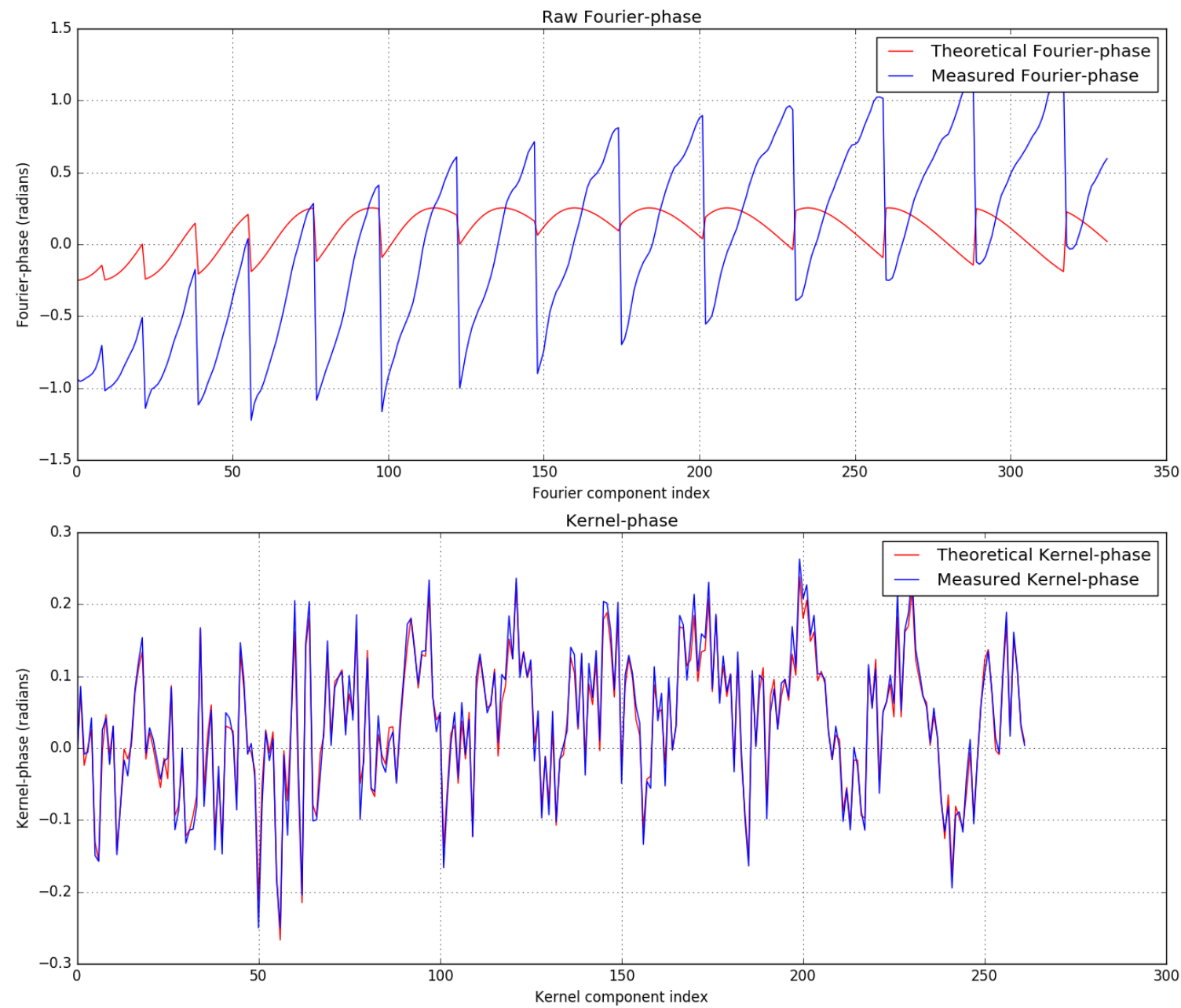

Figure 5. Top: Comparison between expected the theoretical binary signature Fourier-phase (the red curve) and the signal (the blue curve) effectively extracted from the Fourier-plane in the presence of instrumental phase error. Bottom: Similar comparison, this time after multiplication of the Fourier-phase vector by the Kernel operator, using the same color code.

To illustrate the interest of this kernel operator, Fig. 4 offers an example of simulated monochromatic image affected by $140 \mathrm{~nm}$ RMS instrumental aberration phase screen. In the Fourier space, represented in the right panel of Fig. 4, the expected binary signature in the phase (a sinusoidal modulation along the direction of the position angle of the binary) is completely washed out by the spurious signature of the instrumental phase. Yet, although is clearly dominates the Fourier space, this instrumental phase error is sufficiently weak to validate the linear hypothesis. One can therefore apply the left-hand side operator $\mathbf{K}$ that was just described to the model given in Eq. 3, that therefore verifies:

$$
\mathbf{K} \cdot \boldsymbol{\Phi}=\mathbf{K} \cdot \Phi_{\mathbf{o}}
$$

The left-hand side multiplication by the Kernel operator produces a reduced number of linear combinations of the Fourier-phase that are expected to be insensitive to instrumental phase aberrations. Fig. 5 offers a graphical demonstration of the impact of the Kernel operator by comparing both the raw Fourier-phase signal and its Kernel, to the expected theoretical signature of the binary target present in the simulated dataset.

Notwithstanding the fact that the raw Fourier phase signal looks nothing like the expected signal, if one blindly compares the standard deviation between the measured and expected raw Fourier-phase $\left(\sigma_{\text {raw }}=0.6\right.$ 

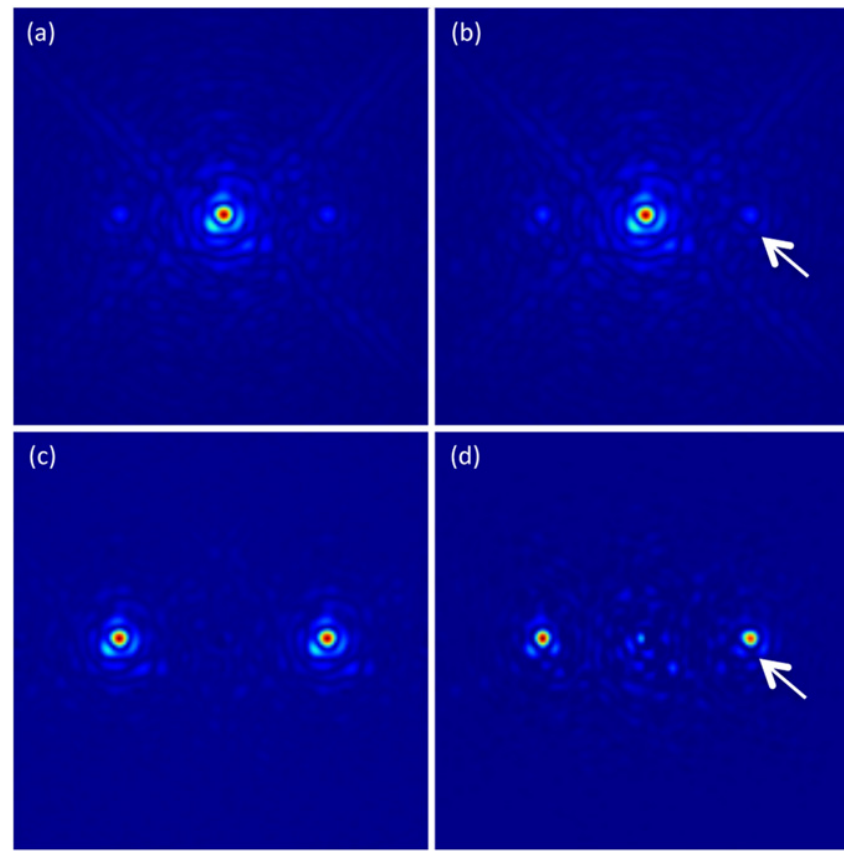

Figure 6. Example of off-axis PSF replicas produced by a sinusoidal modulation of SCExAO's deformable mirror at angular separation $10 \lambda / D$. Images at the bottom are with the on-axis PSF subtracted. Images in panels (a) and (c) in the left column benefit from the dynamic phase modulation that renders them incoherent with the underlying speckle field. Images in panels (b) and (d) in the right column result from a static modulation. The reader will observe that in the static case, the general shape off-axis replicas on the right and left can differ from the on-axis PSF. The dynamic modulation renders these speckles faithful replicas of the on-axis PSF.

radians) to that between the measured and expected kernel-phase ( $\sigma_{\text {raw }}=0.03$ radians), the benefit of the Kernel operator is made obvious: at the cost of a $\sim 20 \%$ reduction in the total number of observable quantities, one goes (for this specific example) from 332 essentially useless raw phases to 262 high-quality observables, that could be reliably used to constrain the properties of the observed source, for instance in a model-fit procedure. On-going efforts are undertaken to improve the phase transfer model and reduce the kernel-phase standard deviation. In their current form, kernels have been successfully been extracted and used from data acquired both by space-borne $\overline{7}[8]$ and AO-corrected ground-based ${ }^{9}$ instruments.

\section{LIMITATIONS AND EXPANSION STRATEGIES}

While remarkably successful already, the Kernel framework has so far been limited by several strict constraints that considerably simplify the interferometric interpretation of existing datasets. As it stands, the framework requires the data to exhibit: a moderate or high-Strehl ( $40 \%)$, a good image sampling (Nyquist or better), and no saturation.

Space borne telescopes able to observe over a wide range of wavelengths usually cannot provide Nyquistsampled data at all wavelengths: sampling can therefore become a real issue in such cases. Most relevant ground based instruments do offer sampling that is compatible with the analysis and provide sufficient material to work from. The other two constraints of non-saturation and capture range are more severe and are the object of theoretical studies and experimental work as part of the KERNEL project.

\subsection{Saturation: kernel-phase with a coronagraph?}

The linear Fourier-phase model operates under the assumption that the instrument response function is truly translation invariant. When attempting the detection of companions of higher contrasts, one eventually confronts the limitation of the dynamical range of the detector. The smallest amount of saturation makes the instrument 
response no-longer truly translation-invariant, and the Fourier-analysis of images no-longer applicable. Yet, the issue can be circumvented by interpolation and/or more advanced reconstruction schemes of the saturated regions of the image, so as to exploit the low spatial frequency (high angular resolution) information available in the non-saturated regions of the images. An extreme use of such extension would be to find the way to produce self-calibrating observable interferometric quantities while taking advantage of the high-contrast boost provided by a coronagraph. By design, a high-contrast coronagraph or nuller tends to destroy the reference PSF leaving very little light available for the extraction of calibrating interferometric observables like kernel-phase.

A solution to this problem lies with the adaptation of a calibration trick used by high-contrast instruments that employs a diffractive element in the pupil to create several off-axis copies of the PSF of adjustable properties. Sinusoidal modulations of a deformable mirror of adjustable frequency and amplitude can be used to produce at specific locations for a given contrast, replicas of the on-axis bright source before it is affected by either saturation or by the presence of a coronagraphic mask. These sinusoidal DM modulations, sometimes also refered to as waffle modes however produce replicas that can interfere with speckles present in the field and produce impredictable results. They can be further improved to be better suited to the needs of the Fourier analysis: rapid alternance between two values of the phase of these waffles $\left(\phi_{o}\right.$ and $\left.\phi_{o}+\pi\right)$ can be used to render them incoherent 10 Fig. 6 is extracted from this reference and presents one example of experimental incoherent speckles produced using this modulation trick. These off-axis reference PSFs of adjustable position and contrast can then reliably be used to recover the information on the PSF that is lost by either saturation or the coronagraph. They can also be used for interferometric wavefront sensing purposes or for local kernel-phase analysis.

\subsection{Capture range}

To remain valid, the linearization described by Eq. 2 requires the phase aberration RMS to be kept small in comparison to the wavelength, limiting the direct application of the KERNEL framework to reasonably well AO-corrected images with Strehl $>40 \%$. The baseline mapping model encoded in the linear operator A however remains perfectly valid and could still be used to produce kernel-phases, if one had access to reliable non-degenerate measurements of the Fourier-phase.

For even in the case of non-redundant baselines, optical path differences (refered to as pistons) larger than the wavelength leads the $2 \pi$-periodic phase to wrap up, rendering the actual phase retrieval degenerate. To resolve this degeneracy, one can exploit the chromatic properties of the instrumental phase: a piston $p_{k}$ (expressed in the same unit as the wavelength) on the baseline of index $k$ introduces a chromatic phase that, assuming that the refraction index of the medium introducing the piston is constant, follows a simple law:

$$
\varphi_{k}(\lambda)=2 \pi p_{k} / \lambda
$$

By monitoring the evolution of the Fourier-phase over a chosen bandpass with a pre-defined spectral resolution, the piston capture range is no longer bound by the wavelength of operation $(p<<\lambda)$, but by the coherence length $\left(p<<\Lambda_{0}\right)$, that can be adapted when adjusting the spectral resolution!11

Figure 7 features one possible form of analysis of the evolution of the complex amplitude measured by a $\mathrm{R}=11$ times redundant baseline in the Fourier plane, by a system providing a uniform sampling between the wavelength 1 and $2 \mu \mathrm{m}$ with a resolution 40. The Fourier analysis of this complex signal makes it possible to detect 11 random piston terms contributing to this signal, as long as they are contained within the coherence length $\Lambda_{0}=R \times \lambda$. Although they cannot be sorted out individually, these pistons can all be measured and added to each other. The result of this sum (multiplied by $2 \pi$ ), can serve as input to the phase transfer model of Eq. 3. in a manner identical to what has been done so far for the Fourier analysis of AO-corrected images:

$$
\phi^{j}=\phi_{0}^{j}+\frac{2 \pi}{R} \sum_{k=1}^{R} p_{k} .
$$

One will notice that unlike Eq. 2 that is a linearized approximation, this new entry is now an exact form. The baseline mapping model whose use has so far been restricted to well corrected images (Strehl better than 


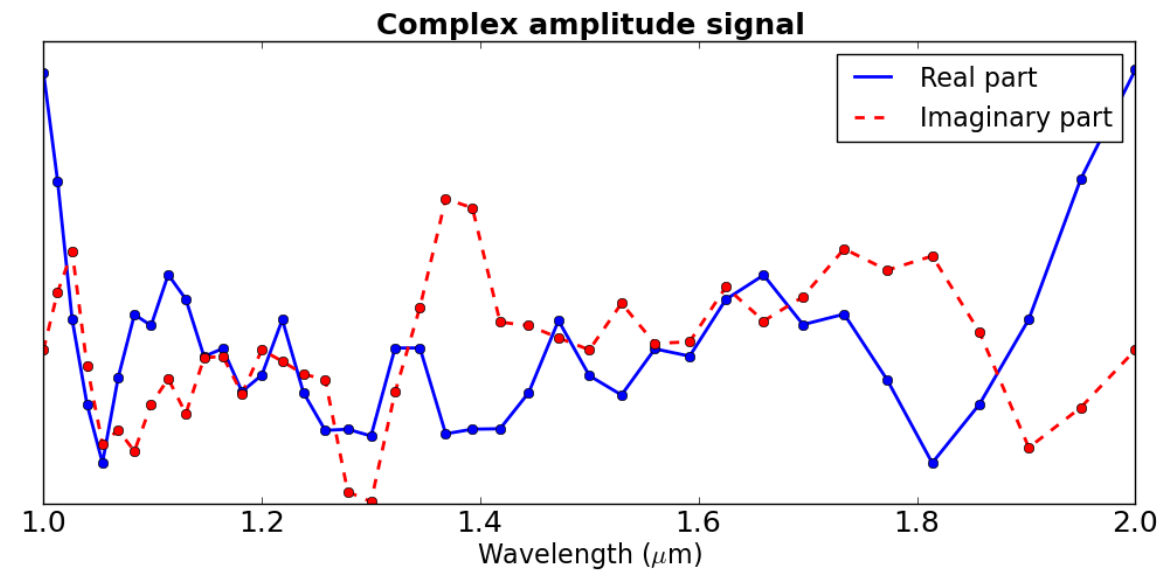

Signal Fourier transform

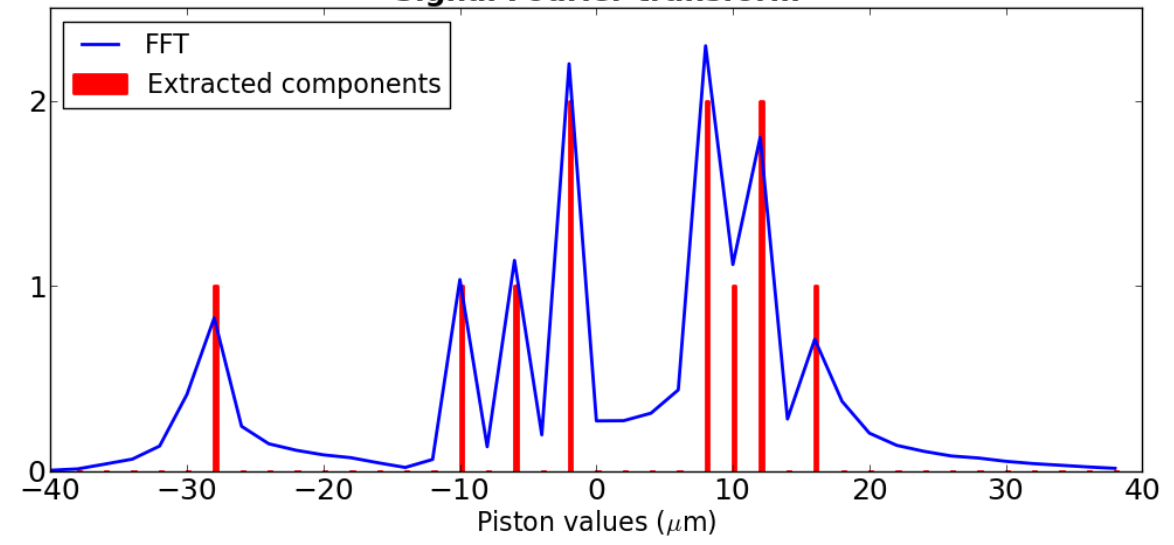

Figure 7. Example of piston extraction for a redundant $(R=11)$ baseline. The fluctuations of the complex amplitude, measured for a given spatial frequency as a function of wavelength (top panel), can be related to the different values of the corresponding 11 pistons, whose values are given by the position of a peak in the Fourier transform of the signal (bottom panel). In this example, 8 distinct peaks are visible. Several baselines indeed feature the same piston, resulting in several Fourier peaks of amplitude twice that of the others. Although the different pistons cannot directly be attributed to baselines, one can add and manipulate them like the linearized phases. 
$\sim 40 \%$ ) remains perfectly valid regardless of the aberration regime. Depending on the specifics of the pupil, one can therefore again either use this composite observable for science (building up kernel-phases) or for wavefront sensing (recovering the full map of piston in the case of an asymmetric pupil), for a naturally redundant aperture.

Other studies ${ }^{12}$ have proposed to exploit the spectral dependance of the piston-induced Fourier-phase information using non-redundant aperture masks to iteratively cophase a segmented telescope like the JWST used as an example. JWST is not equiped with an integral field spectrograph that would otherwise provide a continuous spectral coverage like the one being hinted at here. Its instruments however employ a collection of spectral filters that can be used to constrain the problem of phase retrieval even if the starting point features aberrations larger than their individual wavelength as demonstrated in prior work. Given the extension of the baseline mapping outlined in this work, an adapted combination of non-redundant masking interferograms ${ }^{13}$ like can be acquired with NIRISS ${ }^{14}$ and conventional (unmasked aperture) images in a finite set of spectral filters provides all that is required to first cophase and maintain the cophasing of a segmented telescope like the JWST. If images at multiple wavelengths are available, low-aberration won't stay much longer among the requirements of the technique.

\section{CONCLUSION}

KERNEL is a project that aims at exploiting the full angular resolution potential of large telescopes. Its framework establishes a bridge between the common interpretation techniques of AO-corrected images and the techniques of long-baseline interferometry, hence bringing super-resolution potential to imaging telescopes. Even if this paper mostly touched on the first application, the KERNEL framework can be used both for the production of clean interferometric observables called kernel-phases for astrophysical purposes or for fine metrology also carried out from the focal plane. Post-processing of available archival data is possible today, assuming that the data respects three fundamental requirements of: sampling (Nyquist or better), mid-to-high-Strehl ratio (40\% or better) and non-saturation.

As described in this communication, ongoing investigations suggest possible ways to bypass the framework's initial limitations: using either multi-wavelength information to break the low-aberration limit, or the diffractive adaptive properties of deformable mirrors to bypass the non-saturation requirement. The complete implementation of these ideas is going to open new possibilities: from the extraction of self-calibrating observables in high-contrast coronagraphic images, to an adaptive optics system only fed by a focal plane camera.

The KERNEL analysis and exploitation software XARA (eXtreme Angular Resolution Astronomy), along with a select collection of tools, are available for download as a python package that is regularly updated to to integrate the tricks and techniques developed as part of the KERNEL project http://github.com/fmartinache/ xara.

\section{ACKNOWLEDGMENTS}

This project has received funding from the European Research Council (ERC) under the European Union's Horizon 2020 research and innovation program (grant agreement CoG-683029).

\section{REFERENCES}

[1] Kraus, A. L. and Ireland, M. J., "LkCa 15: A Young Exoplanet Caught at Formation?," ApJ 745, 5 (Jan. 2012).

[2] Biller, B., Lacour, S., Juhász, A., Benisty, M., Chauvin, G., Olofsson, J., Pott, J.-U., Müller, A., SiciliaAguilar, A., Bonnefoy, M., Tuthill, P., Thebault, P., Henning, T., and Crida, A., "A Likely Close-in Low-mass Stellar Companion to the Transitional Disk Star HD 142527," ApJ 753, L38 (July 2012).

[3] Guyon, O. and Martinache, F., "How Extremely Large Telescopes (ELTs) will Acquire the First Spectra of Rocky Habitable Planets," in [American Astronomical Society Meeting Abstracts \#221], American Astronomical Society Meeting Abstracts 221, 419.05 (Jan. 2013).

[4] Jennison, R. C., "A phase sensitive interferometer technique for the measurement of the Fourier transforms of spatial brightness distributions of small angular extent," MNRAS 118, 276-+ (1958). 
[5] Baldwin, J. E., Haniff, C. A., Mackay, C. D., and Warner, P. J., "Closure phase in high-resolution optical imaging," Nature 320, 595-597 (Apr. 1986).

[6] Rajagopal, J. K., Barry, R., Lopez, B., Danchi, W. C., Monnier, J. D., Tuthill, P. G., and Townes, C. H., "Adaptive optics and aperture masking: a comparison," in [Society of Photo-Optical Instrumentation Engineers (SPIE) Conference Series], W. A. Traub, ed., Presented at the Society of Photo-Optical Instrumentation Engineers (SPIE) Conference 5491, 1120-+ (Oct. 2004).

[7] Martinache, F., "Kernel Phase in Fizeau Interferometry," ApJ 724, 464-469 (Nov. 2010).

[8] Pope, B., Martinache, F., and Tuthill, P., "Dancing in the Dark: New Brown Dwarf Binaries from Kernel Phase Interferometry," ApJ 767, 110 (Apr. 2013).

[9] Pope, B., Tuthill, P., Hinkley, S., Ireland, M. J., Greenbaum, A., Latyshev, A., Monnier, J. D., and Martinache, F., "The Palomar kernel-phase experiment: testing kernel phase interferometry for groundbased astronomical observations," MNRAS 455, 1647-1653 (Jan. 2016).

[10] Jovanovic, N., Guyon, O., Martinache, F., Pathak, P., Hagelberg, J., and Kudo, T., "Artificial Incoherent Speckles Enable Precision Astrometry and Photometry in High-contrast Imaging," ApJ 813, L24 (Nov. 2015).

[11] Martinache, F., "Spectrally dispersed Fourier-phase analysis for redundant apertures," in [Optical and Infrared Interferometry and Imaging V], Proc. SPIE 9907, 990712 (Aug. 2016).

[12] Cheetham, A. C., Tuthill, P. G., Sivaramakrishnan, A., and Lloyd, J. P., "Fizeau interferometric cophasing of segmented mirrors," Optics Express 20, 29457 (Dec. 2012).

[13] Cheetham, A., Cvetojevic, N., Norris, B., Sivaramakrishnan, A., and Tuthill, P., "Fizeau interferometric cophasing of segmented mirrors: experimental validation," Optics Express 22, 12924 (June 2014).

[14] Sivaramakrishnan, A., Lafrenière, D., Ford, K. E. S., McKernan, B., Cheetham, A., Greenbaum, A. Z., Tuthill, P. G., Lloyd, J. P., Ireland, M. J., Doyon, R., Beaulieu, M., Martel, A., Koekemoer, A., Martinache, F., and Teuben, P., "Non-redundant Aperture Masking Interferometry (AMI) and segment phasing with JWST-NIRISS," in [Space Telescopes and Instrumentation 2012: Optical, Infrared, and Millimeter Wave], Proc. SPIE 8442, 84422S (Sept. 2012). 